\title{
Efficacy of a self-help parenting intervention for parents of children with attention deficit hyperactivity disorder in adjunct to usual treatment-Small-scale randomized controlled trial
}

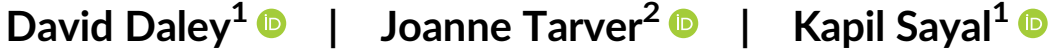

${ }^{1}$ Division of Psychiatry and Applied Psychology, School of Medicine, University of Nottingham, Nottingham, UK

${ }^{2}$ School of Life and Health Sciences, Aston University, Birmingham, UK

\section{Correspondence}

David Daley, Division of Psychiatry and Applied Psychology, School of Medicine, University of Nottingham, Nottingham, UK. Email: david.daley@nottingham.ac.uk

Funding information

National Institute for Health Research (NIHR) Collaborations for Leadership in Applied Health Research and Care-Nottinghamshire, Derbyshire, Lincolnshire (CLAHRC-NDL); Institute of Mental Health at the University of Nottingham

\begin{abstract}
Background: Multimodal intervention incorporating psychosocial intervention and medication is recommended for school-aged children with attention deficit hyperactivity disorder (ADHD). This randomized controlled trial (RCT) investigates the adjunctive benefit of the self-help version of the New Forest Parenting Programme (NFPP-SH) when offered in addition to treatment as usual (TAU) compared with TAU alone.

Method: Fifty-two children, receiving medication for ADHD as part of their usual care, were randomized to receive NFPP-SH + TAU or TAU alone.

Results: When used in adjunct to TAU, NFPP-SH may have beneficial effects for parenting efficacy $(F=6.28, p=0.02)$, child social performance in school and negative comments made by parents during a recorded speech sample. However, the self-help intervention did not have any additional effect on child behaviour.

Conclusions: This study provides further support for self-help interventions as potentially low-intensity and cost-effective alternatives to therapist-led parenting interventions. The findings require replication in larger samples before any firm conclusions about adjunctive efficacy of NFPP-SH can be drawn but underline the potential for self-help within routine treatment (ClinicalTrials.gov Identifier: NCT02174952).

\section{KEYWORDS}

ADHD, behavioural intervention, self-help
\end{abstract}

\section{1 | INTRODUCTION}

Parenting interventions are recommended as part of a multimodal treatment approach for school-aged children with attention deficit hyperactivity disorder (ADHD) (NICE, 2018). Based on social learning principles, parenting interventions include strategies for parents aimed at increasing the frequency of adaptive child behaviours while reducing the occurrence of non-compliant or disruptive behaviour However, their efficacy as treatments for ADHD has been questioned in a meta-analysis that found effect sizes for ADHD symptoms dropped to near zero when using outcome data from objective informants 'probably blind' to treatment allocation (Sonuga-Barke et al., 2013). Indeed, behavioural interventions such as parenting programmes may be better viewed as treatments with the ability to target some of the more distal functioning deficits associated with ADHD (Daley et al., 2014; Sonuga-Barke et al., 2013). This is especially true when behavioural programmes are offered as an adjunct to medication, which is associated with large effect sizes for

This is an open access article under the terms of the Creative Commons Attribution License, which permits use, distribution and reproduction in any medium, 
ADHD symptoms. When analysing data from 'probably blind informants', there is more convincing evidence of the effectiveness of parenting interventions for child conduct problems, parenting behaviour and parenting efficacy (Daley et al., 2014).

There is mixed evidence regarding the additional benefits of parent interventions to medication. The Multimodal Treatment Study for ADHD (MTA) reported no additional benefit of intensive multicomponent behavioural intervention incorporating parent training to medication compared with treatment with medication alone (The MTA Cooperative Group, 1999). However, a reanalysis of MTA data based on the number of children displaying an 'excellent response' to treatment highlighted that $68 \%$ of children receiving multimodal treatment showed an excellent response compared with $56 \%$ of those receiving medication alone (Swanson et al., 2001). There is also evidence of additional benefits for externalizing and internalizing child symptoms when a parenting intervention is added to treatment with medication alone (van den Hoofdakker et al., 2007). When offered as a stand-alone treatment, parenting interventions have beneficial effects for parental well-being including parenting efficacy and parental low mood (Daley \& O'Brien, 2013; Hoath \& Sanders, 2002; Sonuga-Barke, Daley, Thompson, Laver-Bradbury, \& Weeks, 2001).

\section{1 | Self-help parenting programmes}

Despite potential family-wide benefit, a number of practical and psychological barriers can limit the availability, uptake and adherence to therapist-led parenting programmes (Prinz \& Sanders, 2007). First, parenting programmes are expensive, and service provision may be limited (Foster, Johnson-Shelton, \& Taylor, 2007). Second, psychological barriers such as perceived stigma or feelings of isolation can also impact on parental willingness to attend sessions (Koerting et al., 2013; Prinz \& Sanders, 2007). Third, parents may experience practical obstacles such as transport or childcare issues that prevent them from being able to attend sessions (Owens et al., 2002).

Consequently, there is growing interest in the development and efficacy of self-help (SH) parenting interventions that have the potential to overcome barriers to take-up or adherence. $\mathrm{SH}$ interventions provide parents with materials that enable them to teach intervention components to themselves with little or no therapist support. Similar to parent-led intervention, $\mathrm{SH}$ interventions have beneficial effects for parent-reported child behaviour and other family-wide outcomes including parental low mood and stress, parenting behaviour and parenting efficacy (Tarver, Daley, Lockwood, \& Sayal, 2014). There is also evidence of $\mathrm{SH}$ treatment effects being maintained at 1 year post-intervention (Ise, Kierfeld, \& Döpfner, 2015). SH parenting interventions therefore have potential to provide a potentially costeffective, low-intensity alternative to therapist-led interventions that can be added to medication to provide a treatment package that adheres to guidelines recommending multimodal intervention.

Few studies have investigated the adjunctive benefit of an $\mathrm{SH}$ parenting intervention to medication for the treatment of ADHD. Long, Rickert, and Ashcraft (1993) provided bibliotherapy to families

\section{Key messages}

- Parent interventions are recommended as a first-line treatment for attention deficit hyperactivity disorder (ADHD), yet a number of practical and psychological barriers can impact their accessibility.

- Self-help parent interventions may provide an accessible alternative to therapist-led parent interventions.

- In this small-scale randomized controlled trial (RCT), receipt of the self-help version of the New Forest Parenting Programme (NFPP), in adjunct to usual treatment, had beneficial effects for parenting efficacy, child social performance in school and negative comments made by parents during a recorded speech sample compared with TAU alone. However, the self-help intervention did not have any additional effect on child behaviour.

- Future research is needed to replicate these findings in a larger sample of children with ADHD.

with a child with a clinical diagnosis of ADHD and receiving medication as part of their usual care. At post-intervention, children in the intervention group scored lower on parent-reported and teacherreported measures of oppositional behaviour. However, there was no difference between groups on parent-reported measures of hyperactivity or impulsivity (Long et al., 1993). More recently, Dose et al. (2017) have found evidence of telephone-assisted SH having additional benefit for teacher-reported ODD symptoms and negative parenting behaviour. These studies provide preliminary support of the potential of $\mathrm{SH}$ interventions for aspects of parent and child well-being when children are receiving medication for ADHD symptoms; these findings now require replication with varying forms of SH intervention (Dose et al., 2017).

\subsection{The New Forest Parenting Programme}

The New Forest Parenting Programme (NFPP) is a parenting intervention developed specifically for the treatment of ADHD (Sonuga-Barke, Thompson, Abikoff, Klein, \& Brotman, 2006). In addition to behaviour management strategies, the intervention includes ideas for games and strategies that target some of the self-regulatory and cognitive deficits often present in ADHD. Therapist-led NFPP has been shown to be effective for the treatment of preschool ADHD and behaviour problems in randomized controlled trials (RCTs) (SonugaBarke et al., 2001; Thompson et al., 2009).

An SH version of NFPP (NFPP-SH) has been developed and trialled in a small-scale study with 43 children aged between 4 and 11 years and meeting diagnostic criteria for ADHD (Daley \& O'Brien, 2013). NFPP-SH was associated with reductions in parentreported ADHD symptoms. However, independent observations of child behaviour failed to confirm this effect. NFPP-SH was also 
associated with large increases in parenting efficacy and satisfaction. NFPP-SH may not be sufficient to treat ADHD alone. Nonetheless, it could be a useful adjunct to medication with potential to combat some of the more distal problems commonly associated with ADHD that medication may be less able to improve.

This study reports the findings of a trial exploring the efficacy of NFPP-SH when used in adjunct to treatment as usual including pharmacotherapy. The trial was designed as a pragmatic trial; few inclusion/exclusion criteria were applied in order to ensure the applicability of the findings to real-world clinical settings.

\section{2 | METHOD}

\subsection{Participants and recruitment}

This study received ethical approval from the NHS Research Ethics Committee (REC Ref: 12/EM/0200). Fifty-two children aged between 6 and 10 years (mean 8.43 years, SD 1.31 years) were recruited from 11 participating community paediatric and child and adolescent mental health clinics throughout England. All participants provided informed written consent. The characteristics of the sample are presented in Table 1. As a pragmatic trial, few exclusion criteria were applied for study eligibility. Children aged 6-10 years were eligible for the trial if they had received a clinical diagnosis of ADHD (confirmed by referring clinician) or were in receipt of medication for ADHD and their parent/caregiver was aged 18 years or over. There were no restrictions on the type of medication children were receiving (e.g. methylphenidate and lisdexamfetamine) or the length of time children had been receiving medication. Children were excluded if their parents were unable to read English (due to copyright restrictions, the SH manual was only available in English) or if the referring clinician felt that the parent/caregiver would be unable to complete the $\mathrm{SH}$ intervention (e.g. parent had severe mental illness). See Figure 1 for the flow of participants through the trial.

\section{2 | Trial design}

Families were randomized to receive NFPP-SH in adjunct to their usual treatment (NFPP-SH + TAU) or to usual treatment alone (TAU) by a member of the Clinical Trials Unit at the University of Nottingham.

Outcome measures were collected at pre-intervention (T1), post-intervention (T2; 12 weeks) and longer term follow-up (T3; 28 weeks). Data were collected via questionnaire batteries sent to parents. Questionnaire batteries also included child report questionnaires to be completed by the child, at home, where possible. Parents were asked to complete the questionnaires and return them to the research team in a prepaid envelope. Five-Minute Speech Samples (FMSS; to provide a measure of parental expressed emotion [EE]) were recorded via the telephone at T1 and T2. Teacher report questionnaires were sent to teachers at $\mathrm{T} 1$ and $\mathrm{T} 2$ to be completed and returned to assess generalization of treatment effects across settings. As parents were encouraged
TAB LE 1 Baseline characteristics of study sample $(n=52)$

\begin{tabular}{|c|c|}
\hline Child age (years), mean (SD) & $8.43(1.31)$ \\
\hline Child gender (male), $n$ (\%) & $44(85 \%)$ \\
\hline White British, $n$ (\%) & 49 (94\%) \\
\hline \multicolumn{2}{|l|}{ Co-morbid disorders (clinical diagnosis), $n(\%)^{a}$} \\
\hline Autism spectrum disorder & $6(12 \%)$ \\
\hline Learning difficulty & $2(4 \%)$ \\
\hline Dyslexia & $2(4 \%)$ \\
\hline Anxiety disorder & $1(2 \%)$ \\
\hline Attachment disorder & $1(2 \%)$ \\
\hline Disruptive behaviour disorder & $2(4 \%)$ \\
\hline Tourette's syndrome & $1(2 \%)$ \\
\hline Parent/main caregiver age (years), mean (SD) & $36.77(6.41)$ \\
\hline Parent/main caregiver gender (female), $n$ (\%) & $50(96 \%)$ \\
\hline \multicolumn{2}{|l|}{ Parent highest level of education, $n$ (\%) } \\
\hline No qualifications & $8(15 \%)$ \\
\hline Completed school/GCSEs & $17(33 \%)$ \\
\hline Completed college/further education/A levels & $15(30 \%)$ \\
\hline Undergraduate degree/higher education & $2(4 \%)$ \\
\hline Postgraduate degree & $10(19 \%)$ \\
\hline \multicolumn{2}{|l|}{ Family income per year, $n(\%)^{b}$} \\
\hline Less than $£ 10000$ & $14(27 \%)$ \\
\hline Between $£ 10000$ and $£ 40000$ & $27(52 \%)$ \\
\hline Over $£ 50000$ & $7(14 \%)$ \\
\hline Parent living alone, $n$ (\%) & $16(31 \%)$ \\
\hline \multicolumn{2}{|l|}{ Medication status } \\
\hline Methylphenidate & 39 (75\%) \\
\hline Atomoxetine & $3(6 \%)$ \\
\hline Lisdexamfetamine & $2(4 \%)$ \\
\hline Combination short- and long-acting methylphenidate & $7(14 \%)$ \\
\hline $\begin{array}{l}\text { Combination short-acting methylphenidate and } \\
\text { atomoxetine }\end{array}$ & $1(2 \%)$ \\
\hline \multicolumn{2}{|l|}{ Length of time child receiving medication } \\
\hline Less than 1 month & $16(31 \%)$ \\
\hline $1-6$ months & $16(31 \%)$ \\
\hline 7-12 months & $9(17 \%)$ \\
\hline Longer than 13 months & $11(21 \%)$ \\
\hline
\end{tabular}

aParent report; co-morbid diagnoses were not confirmed with referring clinicians.

${ }^{b} n=4$ declined to provide.

to share some aspects of the SH intervention with teachers, teachers were not considered blinded informants in this study.

\section{3 | Treatment arms}

\subsection{1 $\quad$ NFPP-SH + TAU}

Participants allocated to $\mathrm{TAU}+\mathrm{SH}$ received an intervention pack containing copies of the published NFPP-SH book (Laver-Bradbury, 


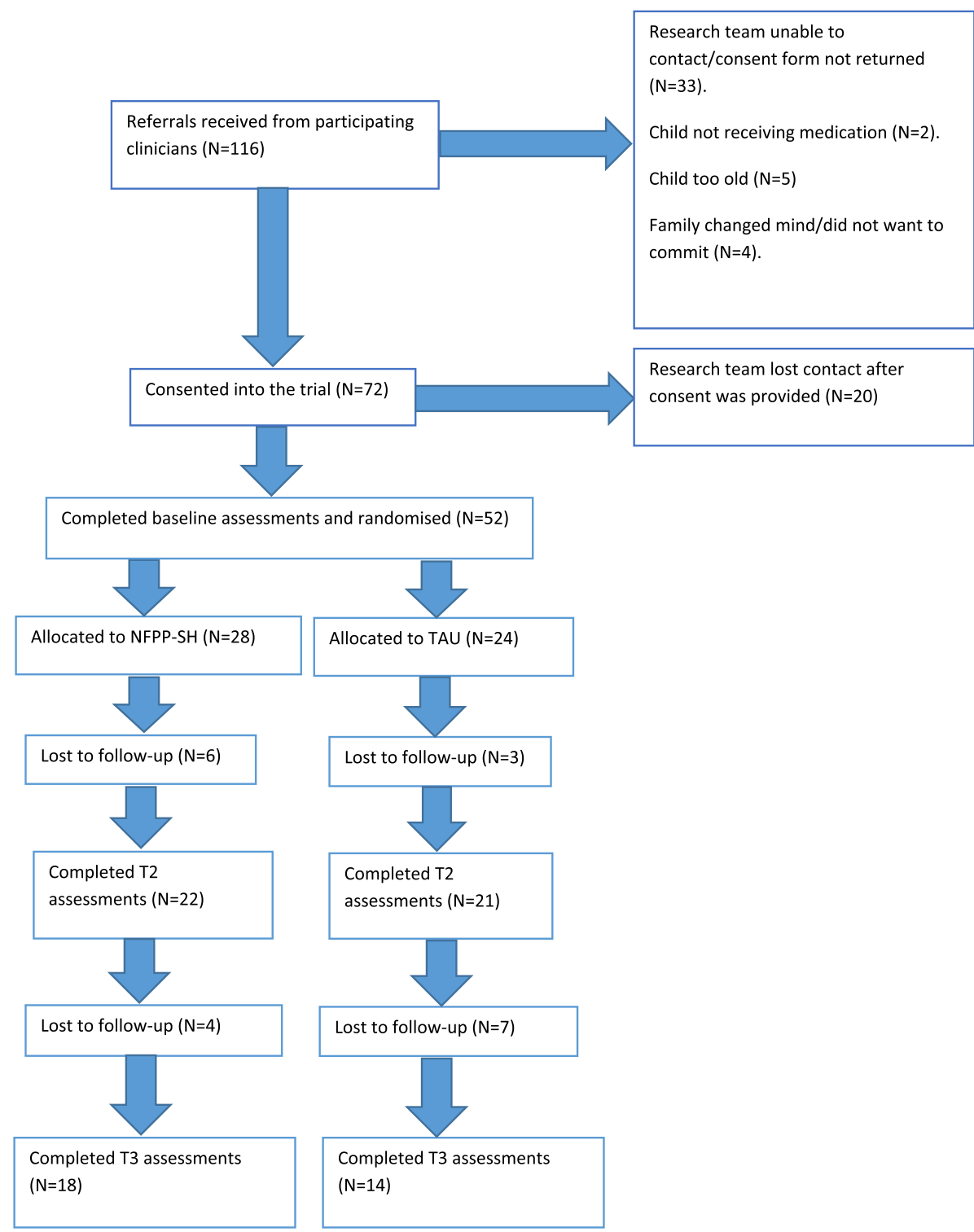

FIGURE 1 CONSORT flow diagram of participant flow through the trial

Thompson, Weeks, Daley, \& Sonuga-Barke, 2010) consisting of two parts. Part 1 includes brief psychoeducation, and Part 2 contains a six-step programme detailing empirically supported behavioural strategies. It was recommended that parents spend 2 weeks reading each step and implementing the strategies. Parents received a fortnightly phone call from a member of university staff external to the research team. The phone call served two purposes: to remind parents to move on the next step of the manual and to collect a measure of $\mathrm{SH}$ treatment fidelity for that fortnight. In the event of an unsuccessful attempt to contact parents by telephone, letters were sent to remind them to move on the next stage of the intervention.

The intervention pack also contained a DVD to accompany the SH manual. The Living with ADHD DVD contained psychoeducation about why children with ADHD behave in the way that they do and also contained a brief summary of the core NFPP strategies that were explained in more detail in the SH book (Laver-Bradbury et al., 2010). Parents received instructions on how to use the DVD in accordance with the SH manual and were advised to share the DVD with others involved in the caregiving of their child (e.g. partners, grandparents and teachers).

\section{\begin{tabular}{l|r} 
2.3.2 & TAU alone
\end{tabular}}

Families allocated to TAU were not contacted by the research team during the 12-week intervention period and received the $\mathrm{SH}$ intervention at the end of their involvement in the trial.

\subsection{Outcome measures}

\subsection{1 | Primary outcome measure: Parenting efficacy}

The Parenting Sense of Competence Scale (PSOC) (Johnston \& Mash, 1989) is a frequently used measure of parenting efficacy 
and satisfaction. The scale has good internal reliability $(\alpha=0.79$, 0.75 and 0.70 for the total scale, satisfaction subscale and efficacy subscale, respectively). The PSOC has been used in previous parenting intervention studies showing sensitivity to treatment effects (e.g. Sonuga-Barke et al., 2001). In this sample, $\alpha=0.58$ and 0.68 for the efficacy and satisfaction subscales, respectively.

\subsection{2 $\quad$ Secondary outcome measures}

\section{Parental mental health}

The 12-item General Health Questionnaire (GHQ-12) (Goldberg, 1982) is a measure of common mental health problems in adults. Respondents rate the presence of each symptom on a 4-point scale (not at all, same as usual, no more than usual, rather more than usual). The scale has good internal reliability $(\alpha=0.91)$ and test-retest reliability (ICC $=0.79)($ Schrnitz, Kruse, \& Tress, 1999). In this sample, the GHQ-12 had an alpha value of 0.89 .

\section{Disruptive behaviour}

The Eyberg Child Behaviour Inventory (ECBI) (Eyberg, Boggs, \& Reynolds, 1980) lists 36 problem behaviours. Parents rate the frequency of each behaviour on a 7-point scale (1 = never and 7 = always; intensity score). Parents rate whether each behaviour is a problem using a 'yes-no' scale (problem score). The reliability of the intensity and problem scales have been demonstrated with mean split-half correlations of $r=0.95$ and 0.94 , respectively (Robinson, Eyberg, \& Ross, 1980). In this sample, the Cronbach's alpha values were 0.90 for the intensity scale and 0.89 for the problem scale.

\section{ADHD and ODD symptoms}

The MTA version of the SNAP-IV (Swanson et al., 2001) contains 26 items measuring hyperactivity/impulsivity ( 9 items), inattention (9 items) and ODD (8 items). Both the parent and teacher versions were employed in this study. Items are rated on a 4-point scale ( 0 = not at all; 1 = just a little; 2 = pretty much; 3 = very much). Bussing et al. (2008) report satisfactory internal reliability for the parent report form ( $\alpha=0.90$ for the inattention subscale, 0.79 for the hyperactivity subscale and 0.89 for the ODD subscale) and teacher report form (0.92 for the inattention subscale, 0.96 for the hyperactivity/impulsivity subscale and 0.92 for the ODD subscale have been reported). Acceptable internal reliabilities were replicated in this sample for the parent $(\alpha=0.80,0.76$ and 0.86 for the inattention, hyperactivity and ODD subscales, respectively) and teacher versions $(\alpha=0.86,0.86$ and 0.83 for the inattention, hyperactivity and ODD subscales, respectively).

\section{Family functioning}

The Family Strain Index (FSI) (Riley et al., 2006) is a six-item measure assessing the impact of ADHD on family experience. Parents are asked to rate the frequency of each item on a 5-point scale ( $0=$ never; 1 = almost never; 2 = sometimes; 3 = almost always; and 4 = always). The scale has demonstrated sensitivity to treatment effects in a previous ADHD treatment study (Svanborg et al., 2009). In this sample, the FSI had a Cronbach's alpha value of 0.85 .

\section{Expressed emotion}

In the FMSS (Daley, Sonuga-Barke, \& Thompson, 2003), parents are asked to talk freely about their thoughts and feelings towards their child. Speech samples are rated on global scales of warmth, relationship and initial statement and frequency counts of positive and negative comments to provide a measure of parental EE. High parental EE is indicated by the presence of a negative rating on one of the global scales or a higher number of negative comments than positive comments (Daley et al., 2003). The measure discriminates between mothers of children with ADHD and mothers of typically developing children (Daley et al., 2003).

\section{Attitudes to drug treatment}

The Southampton ADHD Medication Behaviour and Attitudes Scale (SAMBA) (Harpur, Thompson, Daley, Abikoff, \& Sonuga-Barke, 2008) has parent and child versions, both of which were used in this study. The parent report has 32 items covering seven factors: perceived costs of taking medication, flexibility, resistance, perceived benefits of taking medication, child stigma, parent stigma and parental inconsistency, with Cronbach's alpha values of $0.83,0.82,0.82,0.81,0.79$, 0.75 and 0.67 , respectively (Harpur et al., 2008). The child report version has 16 items containing four factors: stigma, perceived benefits, perceived costs and child's resistance with reported Cronbach's alpha values of $0.82,0.82,0.76$ and 0.79 , respectively. In this study, $\alpha=0.88,0.81,0.70,0.72,0.78,0.54$ and 0.57 for the benefits, costs, child stigma, parent stigma, flexibility, resistance and parental inconstancy subscales, respectively. Cronbach's alpha values for the child report questionnaire were $0.73,0.68,0.57$ and 0.61 for the benefits, stigma scale, perceived costs and resistance subscales, respectively.

\section{Academic and social functioning}

The performance scale of the Vanderbilt ADHD Diagnostic Parent Rating Scale (Wolraich et al., 2003) is an eight-item scale and was used to provide a brief measure of academic and social functioning. Parents/teachers rate the child's performance on a 5-point scale where 1 = problematic and 5 = above average. In this sample, the questions relating to academic performance on the parent report version had a Cronbach's alpha value of 0.84 , whereas the questions relating to child social performance had a Cronbach's alpha value of 0.70 .

\section{Health-related quality of life}

The child report form of the Child Health and Illness Profile (CHIP$\mathrm{CE} / \mathrm{CRF}$ ) is 45-item measure of health-related quality of life (HRQOL) for children (Riley et al., 2004a). It has five scales measuring satisfaction (with health), comfort, resilience, risk avoidance and achievement. Each scale has good internal reliability with Cronbach's alpha values of $0.81,0.82,0.70,0.82$ and 0.74 for the satisfaction, comfort, resilience, risk avoidance and achievement scores, respectively (Riley et al., 2004a). In this sample, Cronbach's alpha values were acceptable 
( $\alpha=0.77,0.74,0.76,0.61$ and 0.87 for the comfort, achievement, risk avoidance, resilience and satisfaction domains, respectively).

The 45-item version of the parent report form of the CHIP-CE (CHIP-CE/PRF-45) was also utilized in this study (Riley et al., 2004b). The 45-item CHIP-CE/PRF is a shortened version of the original 76-item questionnaire that has good test-retest reliability $($ ICC $=0.79,0.71,0.80$, 0.84 and 0.85 for the satisfaction, comfort, resilience, risk avoidance and achievement scales, respectively) and internal reliability ( $\alpha=0.84,0.88$, $0.79,0.82$ and 0.83 for the satisfaction, comfort, resilience, risk avoidance and achievement scales, respectively) (Riley et al., 2004b). The acceptable internal reliability of the scales was replicated in this sample ( $\alpha=0.87,0.84,0.77,0.70$ and 0.66 ) for the satisfaction, comfort, resilience, risk avoidance and achievement domains, respectively.

\section{$\mathrm{SH}$ treatment fidelity}

During fortnightly phone calls, parents in the NFPP $+\mathrm{SH}$ were asked to rate their engagement with the $\mathrm{SH}$ materials over the past fortnight. Parents were asked to rate (on a 5-point scale) the amount of reading they have completed that fortnight ( 1 = I have not read any; 2 = I have read a little; $3=$ I have read about half; $4=$ I have read the majority; $5=$ I have read all of the step). Parents were also asked to rate on a similar scale how frequently they engaged in the strategies included in the step for that fortnight. This provided a measure of self-reported treatment fidelity similar to that used by Svanborg et al. (2009).

\section{Current treatment}

At each time point, parents completed a treatment report form detailing the medication that their child is currently receiving (including dosage), how many contacts they have had with their clinician over the last 3 months (phone and face to face) and whether they are currently participating in any other form of behavioural or parenting intervention programme.

\section{5 | Analysis strategy}

All data analyses were conducted using IBM SPSS Version 21.0. Data were analysed using an intention-to-treat approach with missing data replaced using the multiple imputation command within SPSS. Multiple imputation is seen as the most reliable way of dealing with missing values compared with more traditional forms of dealing with missing data such as last observation carried forward (Acock, 2005). In line with recommendations, 40 imputations were run for the analysis, and the findings reported represent the pooled data for the 40 imputation patterns (Graham, Olchowski, \& Gilreath, 2007). The Markov chain Monte Carlo (MCMC) method was used to impute missing data values (Graham, 2012).

Descriptive statistics were used to explore the means and standard deviations for the primary outcome and secondary outcomes; subscale data were also explored where appropriate. Logistic regression was used to explore possible associations with missing data and treatment dropout. Baseline equivalence between treatment groups was analysed using a series of $t$-tests and chi-square tests.
Mann-Whitney $U$ tests were used to explore baseline equivalence on measures that were non-normally distributed.

To assess for differences between groups at T2 and T3, a series of analyses of covariance (ANCOVAs) were conducted with T2 or T3 scores entered as the dependent variable and T1 scores and other potential confounding variables entered as covariates. ANCOVA is robust to the violation of the non-parametric assumption with more than 15 cases per cell. Effect sizes were calculated by dividing change in scores (T1 to T2 and T1 to T3) by the pooled pretest standard deviation (Morris, 2008). The analyses did not apply adjustments for multiple outcomes (e.g. Bonferroni adjustments). As this was the first trial assessing the adjunctive benefit of NFPP-SH, the authors did not want to increase the risk of Type II error.

\section{3 | RESULTS}

\section{1 | Preliminary analyses}

Twenty-eight families were allocated to NFPP-SH, and 24 families were allocated to TAU. Of the 32 children that completed child report questionnaires at baseline (T1), 21 were allocated to NFPP-SH, and 11 allocated to TAU. There were significantly more children who completed questionnaires in the NFPP-SH group $\left(\chi^{2}[1]=4.65, p<0.05\right)$. At baseline, teacher-reported data were available for 15 children in the NFPP-SH arm and 13 children in the TAU arm. There was no difference between the groups on any demographic variables or baseline characteristics.

\subsection{Attrition}

T2 data were missing for nine families giving an overall attrition rate of approximately $17 \%$, a similar rate to the previous trial of the NFPP$\mathrm{SH}$ [8]. Of the nine participants that dropped out, six were in the NFPP-SH treatment arm, and three were in the TAU treatment arm, a non-significant difference $\left.\chi^{2}[1]=0.72, p>0.05\right)$. No differences were identified between those who dropped out of the study and those who did not. Of these who had responded at T1, T2 teacher questionnaires were returned by 25 teachers (89\%), and T2 child report questionnaires were returned by 26 children (81\%).

T3 data were missing for 20 families (38\% attrition; 10 in the NFPP$\mathrm{SH}$ treatment arm and 10 in the TAU treatment arm). Rates of dropout at T3 did not differ significantly between treatment groups $\chi^{2}[1]=0.93$, $p>0.05)$. No significant differences were identified between those who did and did not drop out at T3. Child questionnaires were available for 18 children (11 NFPP-SH and 7 TAU) at T3 (50\%).

\subsection{Intervention effects on parental well-being}

There was no significant effect of treatment on parenting efficacy at T2. Similarly, there was no effect of intervention on parenting 
$\frac{\sqrt{-5}}{5}$

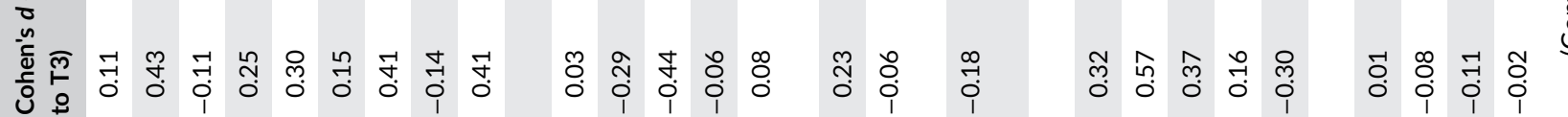

$\underset{\sigma}{E}$

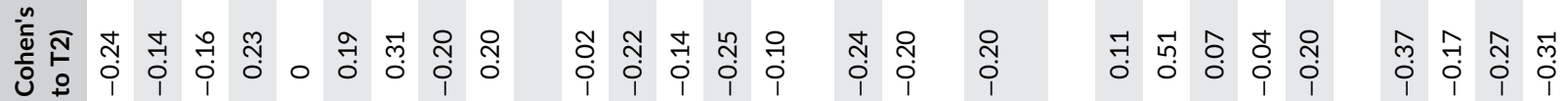

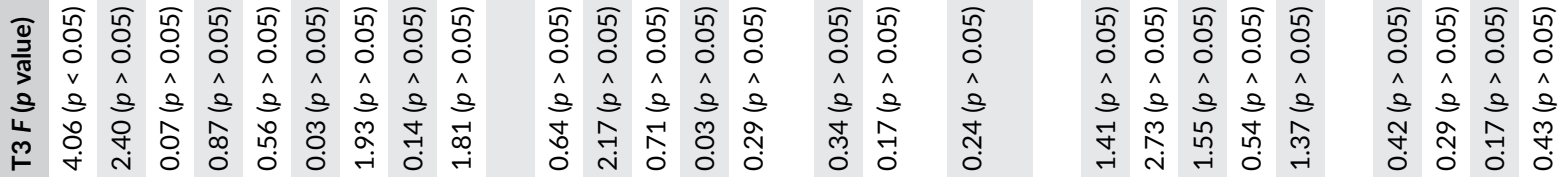

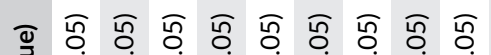

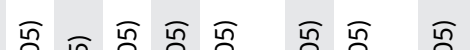

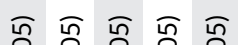

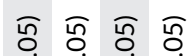

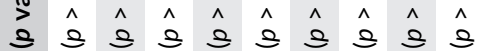

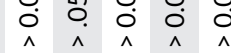

i.

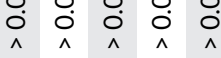

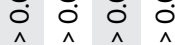

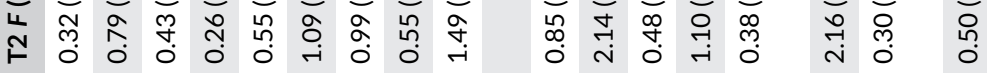

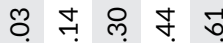

$\hat{\theta} \hat{\theta} \hat{\theta}$

ic

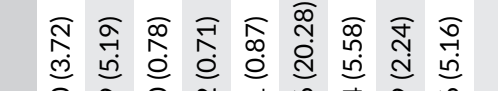

व

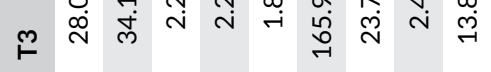

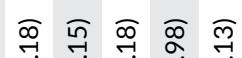

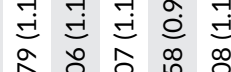

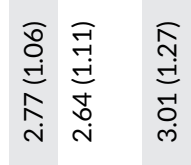

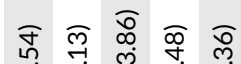

峞

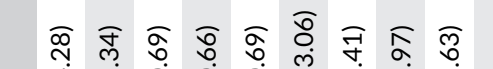

竎

ヘิ

a

लिं 守

ণิ

岕

2 $\stackrel{\substack{0 \\ \infty}}{\infty}$ ते

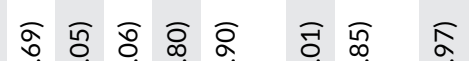

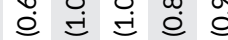

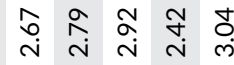

$\stackrel{i}{\mathrm{i}}$

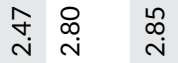

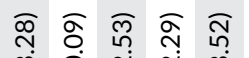

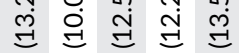

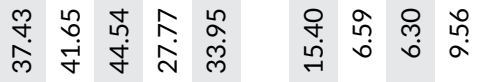

ल ल

œ

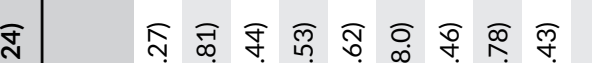
苞

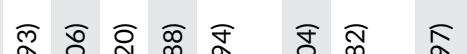

ڤ.

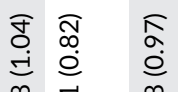

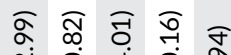

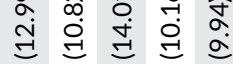

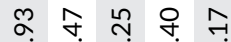

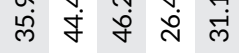

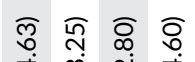

兴

$\neg$ \丶

茟

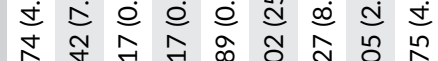

m

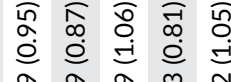

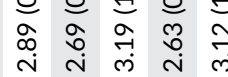

๙ิ

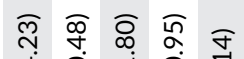

过 लु

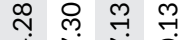

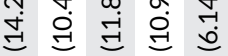

落

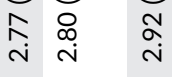

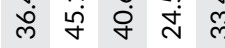

ले वू वิ

ले ले

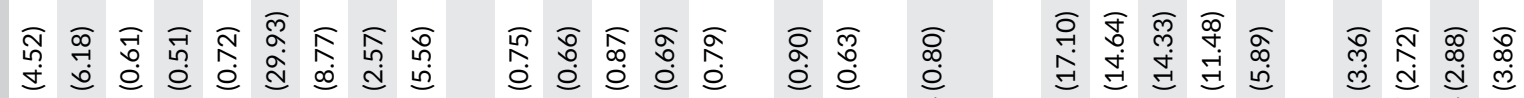

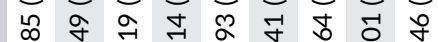

N

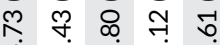

में $\vec{\gamma} \infty$

吾

a d

4 4 ल

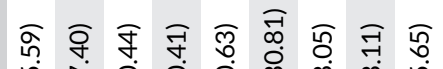

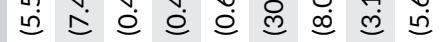

นิ

๙ู. สู

至

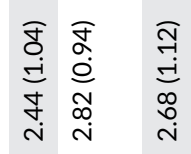

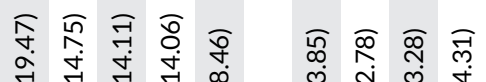

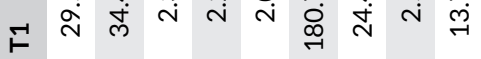

ヘ

ले 命合

ल

ले लि

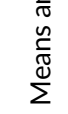

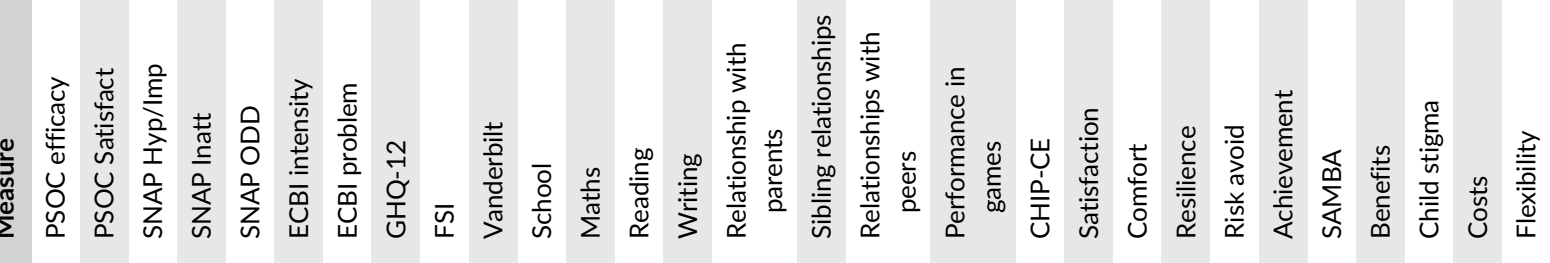




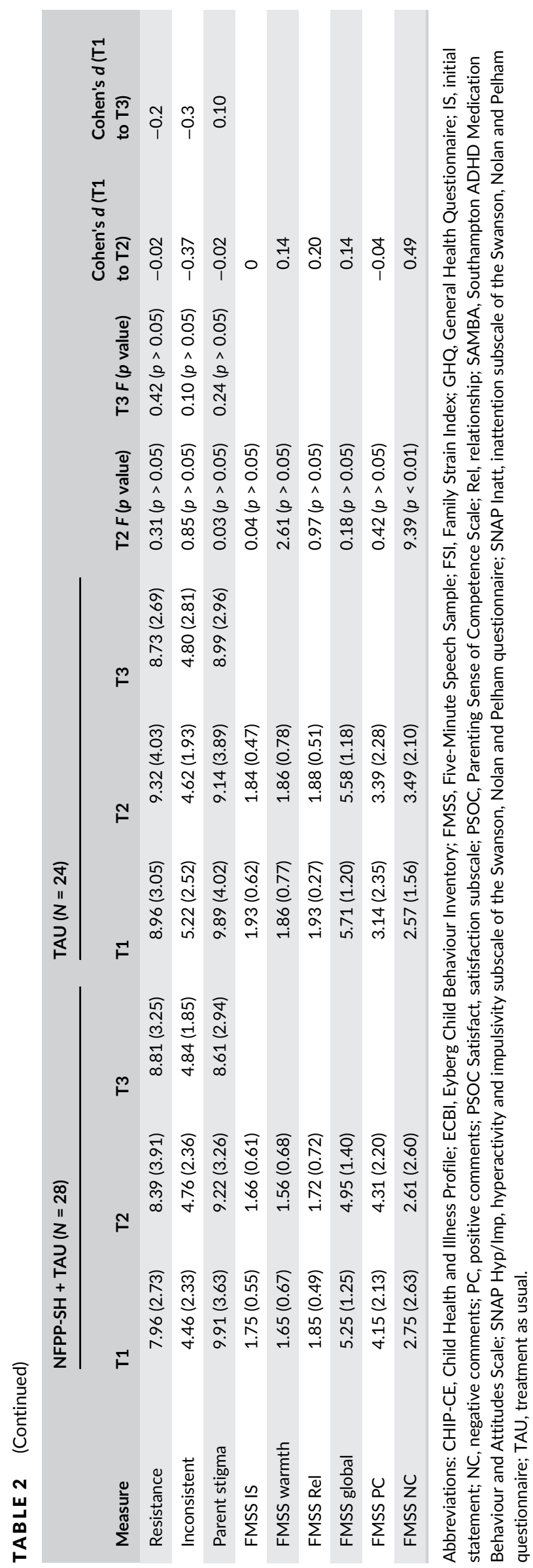

satisfaction or parental mental health at T2. At T3, there was a significant effect of treatment group on parenting efficacy (Table 2; $F$ $[1,49]=4.06, p=0.02$ ). The treatment effect favoured the intervention group but was small in magnitude $(d=0.11)$. There was no effect of intervention on parental mental health or parenting satisfaction at T3.

\subsection{Intervention effects on parent-reported child outcomes}

There were no effects of treatment group on parent-reported child outcomes at T2 or T3 (see Table 2).

\subsection{Intervention effects on family functioning}

There was no significant effect of intervention on family strain according to parent report on the FSI at T2. However, when looking at data for study completers only, the effect of intervention on family strain was significant at T3 $(F[1,29]=5.41, p<0.05)$. Parents who received the $\mathrm{SH}$ intervention and returned $\mathrm{T} 3$ assessments reported lower levels of family strain at T3 compared with families in the TAU who returned T3 assessments (mean 12.50 vs. 14.29).

\section{6 | Treatment effects on parental expressed emotion}

There was a significant effect of treatment group on the number of negative comments that parents made about their child during the FMSS $(F[1,31]=9.39, p<0.01)$. At T2, parents in the NFPP-SH group made fewer negative comments about their child than parents in the TAU control arm after controlling for pretreatment scores. This difference had a moderate effect size $(d=0.49)$. There was no significant effect of treatment on any of the global measures collected in the FMSS or the number of positive comments.

\section{7 | Intervention effects on child-reported outcomes}

There was no significant difference between the treatment groups at T2 or T3 on any of the child-reported CHIP-CE or SAMBA subscales (see Tables S1 and S2).

\section{8 | Intervention effects on teacher-reported outcomes}

There was a significant effect of treatment on teacher reports of the child's relationships with their peers at $\mathrm{T} 2(F[1,26]=6.28, p<0.05)$. Although performance in peer relationships deteriorated in the TAU arm over the 12-week intervention period (mean change $=-0.29$ ), 
teachers reported improvements in peer relationships in children in the NFPP-SH treatment arm (mean change $=0.30$ ); this difference had a large effect size $(d=0.75)$. There were no other significant differences on teacher-reported outcomes at T2 (see Tables S1 and S2).

\section{9 $\quad$ Change in medication status}

Changes in medication status during the intervention period were explored for study completers. There was no difference in medication status change between the two groups at T2 $\left(\chi^{2}[3]=2.73, p>0.05\right)$ or T3 $\left.\chi^{2}[4]=7.20, p>0.05\right)$. The number of parent-reported contacts with clinician did not differ between the groups at T2 $(t[41]=-0.67, p>0.05)$ or T3 $(t[30]=-0.91, p>0.05)$.

\section{4 | DISCUSSION}

This study presents findings from the first RCT of the NFPP-SH when used in adjunct to TAU including pharmacotherapy. The analyses provided some evidence of the adjunctive benefit of the NFPP-SH. Consequently, this low-intensity intervention may have additional beneficial effects for some of the more distal problems commonly associated with ADHD. At T2, parents in the NFPP-SH treatment arm made fewer negative comments about their child- and teacherreported improvements in peer relationships. At T3, parenting efficacy was higher in the NFPP-SH treatment arm compared with the control arm, albeit with a small effect size. A larger effect size was anticipated given the effect size for parenting efficacy reported in the previous study of the NFPP-SH (Daley \& O'Brien, 2013). It is of note that levels of parenting efficacy were higher than expected at study entry $(29.50$ and 27.30 for the NFPP-SA + TAU and TAU groups, respectively), which may explain the small effect size.

There were no other differences between groups on measures of parental well-being. The findings presented herein are in keeping with a recent meta-analysis that did not find any effect of behavioural interventions on parental well-being (Daley et al., 2014). Again, levels of well-being were high in this sample at baseline, perhaps reflecting those parents who are willing to participate in an RCT of SH behavioural interventions.

The NFPP-SH treatment arm fared better on teacher reports of child performance in peer relationships in school at T2. This finding is particularly striking because previous research has failed to find any adjunctive benefit of a parenting intervention for child social performance (Abikoff et al., 2004). The NFPP-SH includes games and activities aimed at improving turn-taking and listening and organizational skills. Through engaging with their children in such activities, it is possible that these skills may have transferred to school and led to improved teacher-rated performance in peer relationships. However, peer relationships were measured in this study via a single item on the Vanderbilt performance scale; this finding should be replicated using a more stringent, multi-item measure of social performance. Furthermore, this finding may reflect the effects of multiple testing.
Finally, parents in the NFPP-SH treatment arm made fewer negative comments about their child during the FMSS at $\mathrm{T} 2$. This replicates the reductions in negative comments observed by Thompson et al. (2009) after receipt of therapist-led NFPP.

There were no differences between groups on measures of ADHD symptoms at T2 or T3. This is in keeping with other multimodal treatment studies (The MTA Cooperative Group, 1999; van den Hoofdakker et al., 2007) and the previous trial assessing the adjunctive benefit of $\mathrm{SH}$ for the treatment of ADHD (Long et al., 1993). In addition, there were no differences between treatment groups on parent- or teacher-reported measures of disruptive behaviour. In contrast, van den Hoofdakker et al. (2007) reported reductions in child externalizing behaviour following receipt of a parenting intervention in addition to routine care compared with routine care alone. It is possible that a larger sample size would have led to the identification of significant effect sizes for disruptive behaviour in this study; effect sizes for parent ECBI scores and teacher SNAP ODD scores favoured the NFPP-SH treatment arm. Although the effect sizes were small in magnitude (approximately $d=0.2$ ), this effect could be meaningful considering both of the arms in the main trial were actively receiving treatment (Kraemer, 1992). However, this should be considered in light of some effect sizes that also favoured the control group, which may also become significant should the trial be replicated in a larger sample.

Few parenting intervention trials to date have explored the effects of parenting interventions on child HRQOL. A trial of psychoeducation for parents of children with ADHD compared with a parent support and counselling intervention found no differences between the groups on measures of HRQOL (Ferrin et al., 2014). In the current study, non-significant effect sizes favoured the intervention group for the majority of HRQOL subdomains, suggesting possible beneficial effects of NFPP-SH for some aspects of HRQOL if the trial were to be replicated in a larger sample.

\section{1 | Methodological considerations}

However, the findings of this trial should be interpreted in the light of some methodological considerations. First, the sample size was small, albeit comparable with other parenting intervention trials in the ADHD literature (Daley \& O'Brien, 2013; Thompson et al., 2009). Although a formal power calculation for the study was not conducted, 52 families may be too few to provide a reliable estimation of treatment effects. Second, the analyses of outcomes in this study did not apply adjustments for multiple outcomes (e.g. Bonferroni adjustments); although the significant effects could be the result of multiple testing, the use of Bonferroni adjustments is controversial, and whereas its use reduces the risk of Type I errors, it does not reduce the risk of Type II errors (Sedgwick, 2012). Third, data collection was reliant on parents completing and returning consent forms and study questionnaires independently. This approach could have led to sample bias because motivated and organized parents may be more likely to return the study questionnaires and consent forms. Indeed, baseline 
levels of parental well-being were higher in this sample than may be anticipated. Fourth, there was very little information available regarding parents use of the $\mathrm{SH}$ manual. Full sets of treatment fidelity scores were only available for six parents. There were insufficient resources in this current study to continue calling parents if they were unable to take the call from the member of the research team collecting the fidelity measures. Because of the levels of missing data, we were unable to explore the relationship between treatment fidelity scores and treatment outcomes. Finally, the overall sample was biased towards a more educated and higher income sample, suggesting that although SH may be helpful, it may not work for all families.

Methodological strengths of this study should also be noted. First, the main trial included outcome measures incorporating measurements of parent and child well-being and family functioning. Assessments of psychosocial intervention should include outcomes where the effects of medication are more uncertain (Antshel \& Barkley, 2008). Second, the study used a multi-informant approach to data collection. Multi-informant approaches including self-report are seen as the gold standard in the assessment of mental well-being. However, it is appreciated that data were missing for teacher and child report questionnaires. Finally, this was a pragmatic trial that aimed to provide an indication of how well the NFPP-SH would work if delivered in real-world clinical settings. Clinical trials lack relevance for clinicians if they exclude patients with co-morbid conditions and test interventions delivered by highly trained, motivated therapists (Glasgow, Magid, Beck, Ritzwoller, \& Estabrooks, 2005). In order to improve the external validity of the findings, few exclusion criteria were applied to the trial.

\section{2 | Implications}

Given the relatively small sample size, it is important for the main trial findings to be replicated in a larger sample that will provide a more reliable analysis of treatment effects. Future research should also include an analysis of cost-effectiveness and include analyses exploring mediators or moderators of treatment outcome. Finally, it would be interesting for future research to have a sample with a broader age range including younger children. Interventions may have superior effects if they are implemented before children are exposed to other potential risk factors including school failure and peer rejection.

\section{ACKNOWLEDGEMENTS}

This study was funded by National Institute for Health Research (NIHR) Collaborations for Leadership in Applied Health Research and Care-Nottinghamshire, Derbyshire, Lincolnshire (CLAHRC-NDL) and the Institute of Mental Health at the University of Nottingham.

\section{CONFLICT OF INTERESTS}

DD is a co-author of the published self-help book that is tested in this study. JT and KS declare no conflicts of interest.

\section{DATA AVAILABILITY STATEMENT}

Full data are not available for ethical reasons.

\section{EDITORIAL OFFICE}

Prof Logan has provided relaxation in the word limit for the manuscript.

\section{ORCID}

David Daley (D) https://orcid.org/0000-0002-3597-0408

Joanne Tarver (D) https://orcid.org/0000-0003-0555-6043

Kapil Sayal (D) https://orcid.org/0000-0002-2050-4316

\section{REFERENCES}

Abikoff, H., Hechtman, L., Klein, R. G., Gallagher, R., Fleiss, K., Etcovitch, J., ... Pollack, S. (2004). Social functioning in children with ADHD treated with long-term methylphenidate and multimodal psychosocial treatment. Journal of the American Academy of Child and Adolescent Psychiatry, 43(7), 820-829. https://doi.org/10.1097/01.chi.0000128797. 91601.1a

Acock, A. C. (2005). Working with missing values. Journal of Marriage and Family, 67(4), 1012-1028. https://doi.org/10.1111/j.1741-3737. 2005.00191.x

Antshel, K. M., \& Barkley, R. (2008). Psychosocial interventions in attention deficit hyperactivity disorder. Child and Adolescent Psychiatric Clinics of North America, 17(2), 421-437, x. https://doi.org/10.1016/j. chc.2007.11.005

Bussing, R., Fernandez, M., Harwood, M., Hou, W., Garvan, C. W., Eyberg, S. M., \& Swanson, J. M. (2008). Parent and teacher SNAP-IV ratings of attention deficit hyperactivity disorder symptoms: Psychometric properties and normative ratings from a school district sample. Assessment, 15(3), 317-328. https://doi.org/10.1177/ 1073191107313888

Daley, D., Sonuga-Barke, E. J. S., \& Thompson, M. (2003). Assessing expressed emotion in mothers of preschool AD/HD children: Psychometric properties of a modified speech sample. The British Journal of Clinical Psychology, 42(Pt 1), 53-67. https://doi.org/10.1348/ 014466503762842011

Daley, D., \& O'Brien, M. (2013). A small-scale randomized controlled trial of the self-help version of the New Forest Parent Training Programme for children with ADHD symptoms. European Child \& Adolescent Psychiatry, 22(9), 543-552. https://doi.org/10.1007/s00787-013-0396-8

Daley, D., van der Oord, S., Ferrin, M., Danckaerts, M., Doepfner, M., Cortese, S., \& Sonuga-Barke, E. J. S. (2014). Behavioral interventions in attention-deficit/hyperactivity disorder: A meta-analysis of randomized controlled trials across multiple outcome domains. Journal of the American Academy of Child \& Adolescent Psychiatry, 53(8), 835-847.e5. https://doi.org/10.1016/j.jaac.2014.05.013

Dose, C., Hautmann, C., Buerger, M., Schuermann, S., Woitecki, K., \& Doepfner, M. (2017). Telephone-assisted self-help for parents of children with attention-deficit/hyperactivity disorder who have residual functional impairment despite methylphenidate treatment: A randomized controlled trial. Journal of Child Psychology and Psychiatry, and Allied Disciplines, 58(6), 682-690. https://doi.org/10.1111/jcpp.12661

Eyberg, S., Boggs, S., \& Reynolds, L. (1980). Eyberg Child Behavior Inventory. University of Oregon Health Sciences Center.

Ferrin, M., Moreno-Granados, J. M., Salcedo-Marin, M. D., RuizVeguilla, M., Perez-Ayala, V., \& Taylor, E. (2014). Evaluation of a psychoeducation programme for parents of children and adolescents with ADHD: Immediate and long-term effects using a blind randomized controlled trial. European Child \& Adolescent Psychiatry, 23(8), 637-647. https://doi.org/10.1007/s00787-013-0494-7 
Foster, E. M., Johnson-Shelton, D., \& Taylor, T. K. (2007). Measuring time costs in interventions designed to reduce behavior problems among children and youth. American Journal of Community Psychology, 40 (1-2), 64-81. https://doi.org/10.1007/s10464-007-9125-0

Glasgow, R. E., Magid, D. J., Beck, A., Ritzwoller, D., \& Estabrooks, P. A. (2005). Practical clinical trials for translating research to practice: Design and measurement recommendations. Medical Care, 43(6), 551-557. https://doi.org/10.1097/01.mlr.0000163645.41407.09

Goldberg, D. P. (1982). General Health Questionnaire manual. NFER.

Graham, J. W. (2012). Missing data: Analysis and design. Springer.

Graham, J. W., Olchowski, A. E., \& Gilreath, T. D. (2007). How many imputations are really needed? Some practical clarifications of multiple imputation theory. Prevention Science: The Official Journal of the Society for Prevention Research, 8(3), 206-213. https://doi.org/10.1007/ s11121-007-0070-9

Harpur, R. A., Thompson, M., Daley, D., Abikoff, H., \& SonugaBarke, E. J. S. (2008). The attention-deficit/hyperactivity disorder medication-related attitudes of patients and their parents. Journal of Child and Adolescent Psychopharmacology, 18(5), 461-473. https://doi. org/10.1089/cap.2008.023

Hoath, F. E., \& Sanders, M. R. (2002). A feasibility study of enhanced group triple P-Positive Parenting Program for parents of children with attention-deficit/hyperactivity disorder. Behaviour Change, 19(4), 191-206. https://doi.org/10.1375/bech.19.4.191

Ise, E., Kierfeld, F., \& Döpfner, M. (2015). One-year follow-up of guided self-help for parents of preschool children with externalizing behavior. The Journal of Primary Prevention, 36(1), 33-40. https://doi.org/10. 1007/s10935-014-0374-z

Johnston, C., \& Mash, E. J. (1989). A measure of parenting satisfaction and efficacy. Journal of Clinical Child Psychology, 18(2), 167-175. https:// doi.org/10.1207/s15374424jccp1802_8

Koerting, J., Smith, E., Knowles, M. M., Latter, S., Elsey, H., McCann, D. C., ... Sonuga-Barke, E. J. (2013). Barriers to, and facilitators of, parenting programmes for childhood behaviour problems: A qualitative synthesis of studies of parents' and professionals' perceptions. European Child \& Adolescent Psychiatry, 22(11), 653-670. https://doi.org/10.1007/ s00787-013-0401-2

Kraemer, H. (1992). Reporting the size of effects in research studies to facilitate assessment of practical or clinical significance. Psychoneuroendocrinology, 17(6), 527-536. https://doi.org/10.1016/ 0306-4530(92)90013-W

Laver-Bradbury, C., Thompson, M., Weeks, A., Daley, D., \& SonugaBarke, E. J. (2010). Step by step help for children with ADHD: A self-help manual for parents. Jessica Kingsley.

Long, N., Rickert, V. I., \& Ashcraft, E. W. (1993). Bibliotherapy as an adjunct to stimulant medication in the treatment of attention-deficit hyperactivity disorder. Journal of Pediatric Health Care, 7(2), 82-88. https://doi.org/10.1016/0891-5245(93)90078-V

Morris, S. B. (2008). Estimating effect sizes from pretest-posttest-control group designs. Organizational Research Methods, 11(2), 364-386. https://doi.org/10.1177/1094428106291059

NICE. (2018). Attention deficit hyperactivity disorder: Diagnosis and management. NICE guideline NG87. The British Psychological Society and the Royal College of Psychiatrist.

Owens, P. L., Hoagwood, K., Horwitz, S. M., Leaf, P. J., Poduska, J. M., Kellam, S. G., \& lalongo, N. S. (2002). Barriers to children's mental health services. Journal of the American Academy of Child \& Adolescent Psychiatry, 41(6), 731-738. https://doi.org/10.1097/00004583200206000-00013

Prinz, R. J., \& Sanders, M. R. (2007). Adopting a population-level approach to parenting and family support interventions. Clinical Psychology Review, 27(6), 739-749. https://doi.org/10.1016/j.cpr.2007. 01.005

Riley, A. W., Forrest, C. B., Rebok, G. W., Starfield, B., Green, B. F., Robertson, J. A., \& Friello, P. (2004a). The child report form of the
CHIP-child edition: Reliability and validity. Medical Care, 42(3), 221-231. https://doi.org/10.1097/01.mlr.0000114910.46921.73

Riley, A. W., Forrest, C. B., Starfield, B., Rebok, G. W., Robertson, J. A., \& Green, B. F. (2004b). The parent report form of the CHIP-child edition: Reliability and validity. Medical Care, 42(3), 210-220. https://doi.org/ 10.1097/01.mlr.0000114909.33878.ca

Riley, A. W., Lyman, L. M., Spiel, G., Döpfner, M., Lorenzo, M. J., Ralston, S. J., \& ADORE Study Group. (2006). The Family Strain Index (FSI). Reliability, validity, and factor structure of a brief questionnaire for families of children with ADHD. European Child \& Adolescent Psychiatry, 15 Suppl, 1, 172-178. https://doi.org/10.1007/s00787-0061010-0

Robinson, E. A., Eyberg, S. M., \& Ross, A. W. (1980). The standardization of an inventory of child conduct problem behaviors. Journal of Clinical Child Psychology, 9(1), 22-28. https://doi.org/10.1080/ 15374418009532938

Schrnitz, N., Kruse, J., \& Tress, W. (1999). Psychometric properties of the General Health Questionnaire (GHQ-12) in a German primary care sample. Acta Psychiatrica Scandinavica, 100(6), 462-468. https://doi. org/10.1111/j.1600-0447.1999.tb10898.x

Sedgwick, P. (2012). Multiple significance tests: The Bonferroni correction. BMJ, 344(jan25 4), e509-e509. https://doi.org/10.1136/bmj.e509

Sonuga-Barke, E. J. S., Brandeis, D., Cortese, S., Daley, D., Ferrin, M., Holtmann, M., ... Dittmann, R. W. (2013). Nonpharmacological interventions for ADHD: Systematic review and meta-analyses of randomized controlled trials of dietary and psychological treatments. American Journal of Psychiatry, 170(3), 275-289. https://doi.org/10.1176/appi. ajp.2012.12070991

Sonuga-Barke, E. J. S., Daley, D., Thompson, M., Laver-Bradbury, C., \& Weeks, A. (2001). Parent-based therapies for preschool attention-deficit/hyperactivity disorder: A randomized, controlled trial with a community sample. Journal of the American Academy of Child \& Adolescent Psychiatry, 40(4), 402-408. https://doi.org/10.1097/00004583200104000-00008

Sonuga-Barke, E. J. S., Thompson, M., Abikoff, H., Klein, R., \& Brotman, L. M. (2006). Nonpharmacological interventions for preschoolers with ADHD: The case for specialized parent training. Infants \& Young Children, 19(2), 142-153. https://doi.org/10.1097/ 00001163-200604000-00007

Svanborg, P., Thernlund, G., Gustafsson, P. A., Hägglöf, B., Poole, L., \& Kadesjö, B. (2009). Efficacy and safety of atomoxetine as add-on to psychoeducation in the treatment of attention deficit/hyperactivity disorder: A randomized, double-blind, placebo-controlled study in stimulant-naïve Swedish children and adolescents. European Child \& Adolescent Psychiatry, 18(4), 240-249. https://doi.org/10.1007/ s00787-008-0725-5

Swanson, J. M., Kraemer, H. C., Hinshaw, S. P., Arnold, L. E., Conners, C. K., Abikoff, H. B., ... Wu, M. (2001). Clinical relevance of the primary findings of the MTA: Success rates based on severity of ADHD and ODD symptoms at the end of treatment. Journal of the American Academy of Child \& Adolescent Psychiatry, 40(2), 168-179. https://doi.org/10.1097/00004583-200102000-00011

Tarver, J., Daley, D., Lockwood, J., \& Sayal, K. (2014). Are self-directed parenting interventions sufficient for externalising behaviour problems in childhood? A systematic review and meta-analysis. European Child \& Adolescent Psychiatry, 23(12), 1123-1137. https://doi.org/10.1007/ s00787-014-0556-5

The MTA Cooperative Group. (1999). A 14-month randomized clinical trial of treatment strategies for attention-deficit/hyperactivity disorder. Archives of General Psychiatry, 56(12), 1073. https://doi.org/10.1001/ archpsyc.56.12.1073

Thompson, M. J. J., Laver-Bradbury, C., Ayres, M., Le Poidevin, E., Mead, S., Dodds, C., ... Sonuga-Barke, E. J. S. (2009). A small-scale randomized controlled trial of the revised new forest parenting programme for preschoolers with attention deficit hyperactivity disorder. 
European Child \& Adolescent Psychiatry, 18(10), 605-616. https://doi. org/10.1007/s00787-009-0020-0

van den Hoofdakker, B. J., van der Veen-Mulders, L., Sytema, S., Emmelkamp, P. M. G., Minderaa, R. B., \& Nauta, M. H. (2007). Effectiveness of behavioral parent training for children with ADHD in routine clinical practice: A randomized controlled study. Journal of the American Academy of Child \& Adolescent Psychiatry, 46(10), 1263-1271. https://doi.org/10.1097/chi.0b013e3181354bc2

Wolraich, M. L., Lambert, W., Doffing, M. A., Bickman, L., Simmons, T., \& Worley, K. (2003). Psychometric properties of the Vanderbilt ADHD diagnostic parent rating scale in a referred population. Journal of Pediatric Psychology, 28(8), 559-567. https://doi.org/10.1093/jpepsy/ jsg046

\section{SUPPORTING INFORMATION}

Additional supporting information may be found online in the Supporting Information section at the end of this article.

How to cite this article: Daley D, Tarver J, Sayal K. Efficacy of a self-help parenting intervention for parents of children with attention deficit hyperactivity disorder in adjunct to usual treatment-Small-scale randomized controlled trial. Child Care Health Dev. 2020;1-12. https://doi.org/10.1111/cch.12825 